\title{
Moment-Based Fast Discrete Sine Transforms
}

\author{
J. G. Liu, F. H. Y. Chan, F. K. Lam, and H. F. Li
}

\begin{abstract}
This paper presents a novel approach to compute discrete sine transforms (DST's). By using a modular mapping, DST's are approximated by the sum of a finite sequence of discrete moments. Hence, by extending our earlier technique in computing moments with an adder network only, DST's can also be implemented easily by a systolic array primarily involving additions. The method can be applied to multidimensional DST's as well as their inverses.
\end{abstract}

Index Terms-Discrete sine transforms, fast transformation, moment, systolic array.

D ISCRETE sine transforms (DST's) are widely used in speech coding and image compression.

The $N$-point DST-II is defined by the equations

$$
X(k)=\sum_{n=1}^{N} x(n) \sin (\pi(2 n-1) k / 2 N) \quad 1 \leq k \leq N
$$

where

$$
c_{k}= \begin{cases}1 / \sqrt{N}, & \text { for } k=N \\ \sqrt{2 / N}, & \text { otherwise. }\end{cases}
$$

Let us simplify (1). By using the periodic property and other properties of sine functions, we have

$$
\sin (\pi(2 n-1) k / 2 N)=\sin (i \pi / 2 N)
$$

or

$$
-\sin (i \pi / 2 N) \quad 0 \leq i \leq N, 1 \leq k \leq N .
$$

For every pair of $k$ and $i(i, k=1,2, \cdots, N)$, defining $S(k, i)$ and $s(k, i)$ by

$$
\begin{aligned}
S(k, i)= & \{j \mid \sin (\pi(2 j-1) k / 2 N) \\
& =\sin (i \pi / 2 N) \quad 1 \leq j \leq N\} \\
s(k, i)= & \{j \mid \sin (\pi(2 j-1) k / 2 N) \\
& =-\sin (i \pi / 2 N) \quad 1 \leq j \leq N\}
\end{aligned}
$$

Manuscript received January 21, 2000. This work was supported by the National Science Foundation of China under Grant 69775011 and National Defense Key Laboratory of China under Grant 98JS93.5.2JW0510, and University of Hong Kong research grants. The associate editor coordinating the review of this manuscript and approving it for publication was Prof. R. L. de Queiroz.

J. G. Liu is with the Key Laboratory of State Education Ministry for Image Processing and Intelligent Control, Institute for Pattern Recognition and Artificial Intelligence, Huazhong University of Science and Technology, Huazhong, China (jgliu@mipg.upenn.edu).

F. H. Y. Chan and F. K. Lam are with the Department of Electrical and Electronic Engineering, The University of Hong Kong, Hong Kong.

H. F. Li is with the Department of Computer Science, Concordia University, Montreal, PQ, Canada.

Publisher Item Identifier S 1070-9908(00)06513-5. then defining $x_{k, i}(i, k=0,1,2, \cdots, N-1)$ by

$$
x_{k, i}= \begin{cases}\sum_{j \in S(k, i)} x(j)-\sum_{j \in s(k, i)} x(j) & \\ S(k, i) \cup s(k, i) \neq \phi, & i, k=1,2, \cdots, N \\ 0, & \text { otherwise. }\end{cases}
$$

Thus, by direct substitution, (1) can be rewritten as follows:

$$
X(k)=c_{k} \sum_{i=1}^{N} x_{k, i} \sin (\pi i / 2 N) \quad 1 \leq k \leq N .
$$

For $1 \leq i \leq N-1$

$$
\begin{aligned}
\sin (\pi i / 2 N)= & (\pi i / 2 N)-(\pi i / 2 N)^{3} / 3 ! \\
& +\cdots+(-1)^{p}(\pi i / 2 N)^{2 p+1} /(2 p+1) !+R_{i} \\
R_{i}= & \sin \left(\xi_{i}+(p+2) \pi /\right) \\
& \cdot(\pi i / 2 N)^{2 p+2} /(2 p+2) ! \quad 0<\xi_{i}<\pi i / 2 N .
\end{aligned}
$$

This follows immediately by applying the theorem of extended law of the mean to $\sin (\pi i / N)$, where $R_{i}$ is a Taylor remainder term. Substituting (4) into (3) yields

$$
\begin{aligned}
X(k)= & c_{k} \sum_{i=1}^{N-1} x_{k, i} \sum_{r=0}^{p}(-1)^{r}(\pi i / 2 N)^{2 r+1} / \\
& \cdot(2 r+1) !+c_{k} x_{k, N}+R_{p} \\
= & c_{k} \sum_{r=0}^{p}(-1)^{r} \pi^{2 r+1} /(2 N)^{2 r+1}(2 r+1) ! \\
& \cdot \sum_{i=1}^{N-1} x_{k, i} i^{2 r+1}+c_{k} x_{k, N}+R_{p} \quad 1 \leq k \leq N \\
= & c_{k} \sum_{r=0}^{p} a_{r} m_{k, 2 r+1}+c_{k} x_{k, N}+R_{p}
\end{aligned}
$$

where

$$
\begin{aligned}
a_{r}= & (-1)^{r} \pi^{2 r+1} /(2 N)^{2 r+1}(2 r+1) ! \\
m_{k, 2 r+1}= & \sum_{i=1}^{N-1} x_{k, i} i^{2 r+1} \\
R_{p}= & c_{k} \sum_{i=1}^{N-1} x_{k i} \sin \left(\xi_{i}+(p+1) \pi\right) \\
& \cdot(\pi i / 2 N)^{2 p+2} /(2 p+2) ! \quad 0<\xi_{i}<\pi i / 2 N .
\end{aligned}
$$


If $R_{p}$ is ignored, we have

$$
X(k)=c_{k}\left(x_{k, N}+\sum_{r=0}^{p} a_{r} m_{k, 2 r+1}\right) \quad 1 \leq k \leq N .
$$

The absolute value of the error introduced by overlooking $R_{p}$ is bounded by

$$
\left|R_{p}\right|=\leq\left.\max _{n} x(n)\right|_{c_{k}}(N-1) \pi^{2 p+2} / 2^{2 p+2}(2 p+2) !
$$

From the inequality above, it is clearly shown that $R_{p}$ converges to zero very rapidly and uniformly. For example, for $\max |x(n)| \leq 256,(0 \leq n \leq N-1), N \leq 2048, p=8$

$$
\left|R_{p}\right| \leq 8.32 \times 10^{-9}
$$

This error can satisfy accuracy requirements of most applications. Furthermore, we can prove that the least upper bound of $p$ is not more than $O\left(\log _{2} N / \log _{2} \log _{2} N\right)$ as $N$ tends to infinite [1], [2].

The $N$-point DST-I is defined by the equations

$$
X(k)=\sqrt{\frac{2}{N}} \sum_{n=1}^{N-1} x(n) \sin \frac{k n \pi}{N} \quad 1 \leq k \leq N-1 .
$$

By using the periodic property and other properties of sine functions, we have

$$
\sin (k n \pi / N)=\sin (i \pi / N)
$$

or

$$
-\sin (i \pi / N) \quad 1 \leq i \leq N, 1 \leq k \leq N-1
$$

For every pair of $k$ and $i(i, k=1,2, \cdots, N)$, defining $S(k, i)$ and $s(k, i)$ by

$$
\begin{aligned}
S(k, i)= & \{j \mid \sin (k j \pi / N) \\
& =\sin (i \pi / N) \quad 1 \leq j \leq N-1\} \\
s(k, i)= & \{j \mid \sin (k j \pi / N) \\
& =-\sin (i \pi / N) \quad 1 \leq j \leq N-1\}
\end{aligned}
$$

then defining $x_{k, i}(i, k=0,1,2, \cdots, N-1)$ by

$$
x_{k, i}= \begin{cases}\sum_{j \in S(k, i)} x(j)-\sum_{j \in s(k, i)} x(j) & \\ S(k, i) \cup s(k, i) \neq \phi, & i, k=1,2, \cdots, N \\ 0, & \text { otherwise. }\end{cases}
$$

Thus, by direct substitution, (11) can be rewritten as follows:

$$
X(k)=\sqrt{\frac{2}{N}} \sum_{n=1}^{N-1} x_{k, 1} \sin \frac{i \pi}{N} \quad 1 \leq k \leq N-1 .
$$

The $N$-point DST-III is defined by the equations

$$
X(k)=\sqrt{\frac{2}{N}} \sum_{n=1}^{N-1} C_{n} x(n) \sin \frac{(2 k-1) n \pi}{2 N} \quad 1 \leq k \leq N
$$

where

$$
c_{n}= \begin{cases}1 / \sqrt{N}, & \text { for } n=N \\ \sqrt{2 / N}, & \text { otherwise }\end{cases}
$$

By using the periodic property and other properties of sine functions, we have

or

$$
\sin (\pi(2 k-1) n / 2 N)=\sin (i \pi / 2 N)
$$

$$
-\sin (i \pi / 2 N) \quad 0 \leq i \leq N, 1 \leq k \leq N
$$

For every pair of $k$ and $i(i, k=1,2, \cdots, N)$, defining $S(k, i)$ and $s(k, i)$ by

$$
\begin{aligned}
S(k, i)= & \{j \mid \sin (\pi(2 k-1) j / 2 N) \\
& =\sin (i \pi / 2 N) \quad 1 \leq j \leq N\} \\
s(k, i)= & \{j \mid \sin (\pi(2 k-1) j / 2 N) \\
& =-\sin (i \pi / 2 N) \quad 1 \leq j \leq N\}
\end{aligned}
$$

then defining $x_{k, i}(i, k=0,1,2, \cdots, N-1)$ by

$$
x_{k, i}= \begin{cases}\sum_{j \in S(k, i)} C_{j} x(j) & \\ -\sum_{j \in s(k, i)} C_{j} x(j) & \\ S(k, i) \cup s(k, i) \neq \phi, & i, k=1,2, \cdots, N \\ 0, & \text { otherwise. }\end{cases}
$$

Thus

$$
X(k)=\sum_{n=1}^{N-1} x_{k, i} \sin \frac{i \pi}{2 N} \quad 0 \leq k \leq N .
$$

The $N$-point DST-IV is defined by the equations

$$
\begin{aligned}
X(k)= & \sqrt{\frac{2}{N}} \sum_{n=0}^{N-1} x(n) \sin \\
& \cdot \frac{(2 k+1)(2 n+1) \pi}{4 N} \quad 1 \leq k \leq N-1 .
\end{aligned}
$$

By using the periodic property and other properties of sine functions, we have

$$
\begin{aligned}
& \sin (2 k+1)(2 n+1) \pi / 4 N \\
& \quad=\sin (i \pi / 4 N) \text { or }-\sin (i \pi / 4 N) \text { or } \\
& \cos (i \pi / 4 N) \text { or }-\cos (i \pi / 4 N) \\
& \quad 0 \leq i \leq N, 1 \leq k \leq N-1 .
\end{aligned}
$$

For every pair of $k$ and $i(i, k=1,2, \cdots, N)$, defining $S(k, i)$, $s(k, i), C(k, i), c(k, i)$ by

$$
\begin{aligned}
S(k, i)= & \{j \mid \sin (2 k+1)(2 j+1) \pi / 4 N) \\
& =\sin (i \pi / 4 N) \quad 1 \leq j \leq N-1\} \\
s(k, i)= & \{j \mid \sin (2 k+1)(2 j+1) \pi / 4 N \\
& =-\sin (i \pi / 4 N) \quad 1 \leq j \leq N-1\}
\end{aligned}
$$




$$
\begin{aligned}
C(k, i)= & \{j \mid \sin (2 k+1)(2 j+1) \pi / 4 N \\
& =\cos (i \pi / 4 N) \quad 1 \leq j \leq N-1\} \\
c(k, i)= & \{j \mid \sin (2 k+1)(2 j+1) \pi / 4 N \\
& =-\cos (i \pi / 4 N) \quad 1 \leq j \leq N-1\}
\end{aligned}
$$

then defining $x_{k, i}$ and $y_{k, i}(i, k=0,1,2, \cdots, N-1)$ by

$$
\begin{aligned}
x_{k, i}=\left\{\begin{array}{lc}
\sum_{j \in S(k, i)} x(j) & \\
-\sum_{j \in s(k, i)} x(j) & i, k=0,1,2, \\
S(k, i) \cup s(k, i) \neq \phi, N-1 & \cdots, N \\
0, & \text { otherwise. }
\end{array}\right. \\
y_{k, i}=\left\{\begin{array}{lc}
\sum_{j \in S(k, i)} x(j) & i, k=0,1,2, \\
-\sum_{j \in s(k, i)} x(j) & \cdots, N-1 \\
C(k, i) \cup c(k, i) \neq \phi, & \text { otherwise. } \\
0, &
\end{array}\right.
\end{aligned}
$$

Thus

$$
\begin{aligned}
X(k)= & \sum_{n=1}^{N-1} \boldsymbol{x}_{k, i} \sin \frac{i \pi}{4 N} \\
& +\sum_{n=0}^{N-1} \boldsymbol{y}_{k, i} \cos \frac{i \pi}{4 N} \quad 0 \leq k \leq N-1 .
\end{aligned}
$$

If applied the theorem of extended law of the mean, (13), (16), and (20) can be transformed into the forms of (9). In other words, an additional three forms of DST could also be computed through computation of moments. They have the same error levels and the same convergence features.

Equation (9) contains the formulas of the relationship between DST and moments. According to it, the computation of $X(k)$ requires the generation of the $2 r+1$ th order moments of the transformed data sequence $x_{k, i}$, followed by computing the dot- product of between this and a constant vector $\left(a_{r}\right)$ and an addition with $x_{k, N}$, finally followed by a multiplication with $c_{k}$. The efficiency of the procedure largely depends on the efficiency in deriving the moments. We have presented an approach to moments of computation that involves in just additions and designed systolic arrays implementing the method [3], [4].

The proposed method has several noteworthy advantages. First, most multiplications are replaced by additions, and triangular functions are replaced with simple polynomial functions. Second, the multiplication number for one-dimensional (1-D) DST in our method is $O\left(N \log _{2} N / \log _{2} \log _{2} N\right)$ superior to $O\left(N \log _{2} N\right)$ needed in conventional fast sine transform. Third, the algorithm can be directly realized in the form of a systolic array for real-time applications. Fourth, the method is easily extendible to multidimensional DST's as well as their inverses. The computational complexity for $k$-dimensional DST is $O\left(n^{k}\right)$.

\section{REFERENCES}

[1] J. G. Liu, H. F. Li, F. H. Y. Chan, and F. K. Lam, "A novel approach to fast discrete Fourier transform," J. Parallel Distrib. Comput., vol. 54, pp. 48-58, 1998.

[2] - "Fast discrete cosine transform via computation of moments," $J$. VLSI Signal Process., vol. 19, pp. 257-268, 1998.

[3] F. H. Y. Chan, F. K. Lam, H. F. Li, and J. G. Liu, "An all adder systolic structure for fast computation of moments," J. VLSI Signal Process., vol. 12, pp. 159-175, 1996.

[4] J. G. Liu, F. H. Y. Chan, F. K. Lam, and H. F. Li, "A new approach to fast calculation of moments of 3-D gray level images," Parallel Comput., vol. 26, no. 6, pp. 805-815, 2000. 